\title{
Near-infrared Raman spectroscopy to detect anti- Toxoplasma gondii antibody in blood sera of domestic cats: quantitative analysis based on partial least-squares multivariate statistics
}

\author{
Janaína Duarte \\ Universidade Paulista \\ Rod. Pres. Dutra, km 157,5 \\ 12240-420, São José dos Campos, São Paulo \\ Brazil
}

\author{
Marcos T. T. Pacheco \\ Antonio Balbin Villaverde \\ Universidade Camilo Castelo Branco \\ Núcleo do Parque Tecnológico de São José dos Campos \\ Biomedical Engineering Group \\ Rod. Pres. Dutra, km 138 \\ 12247-004 São José dos Campos, São Paulo \\ Brazil
}

Rosangela Z. Machado

Universidade Estadual Paulista Júlio de Mesquita Filho Department of Veterinary Pathology

Via Paulo Donato Castellane, S/N

14884-900 Jaboticabal, São Paulo

Brazil

Renato A. Zângaro

Landulfo Silveira Jr.

Universidade Camilo Castelo Branco

Núcleo do Parque Tecnológico de São José dos Campos

Biomedical Engineering Group

Rod. Pres. Dutra, km 138

12247-004 São José dos Campos, São Paulo

Brazil

\begin{abstract}
Toxoplasmosis is an important zoonosis in public health because domestic cats are the main agents responsible for the transmission of this disease in Brazil. We investigate a method for diagnosing toxoplasmosis based on Raman spectroscopy. Dispersive nearinfrared Raman spectra are used to quantify anti-Toxoplasma gondii (IgG) antibodies in blood sera from domestic cats. An 830-nm laser is used for sample excitation, and a dispersive spectrometer is used to detect the Raman scattering. A serological test is performed in all serum samples by the enzyme-linked immunosorbent assay (ELISA) for validation. Raman spectra are taken from 59 blood serum samples and a quantification model is implemented based on partial least squares (PLS) to quantify the sample's serology by Raman spectra compared to the results provided by the ELISA test. Based on the serological values provided by the Raman/PLS model, diagnostic parameters such as sensitivity, specificity, accuracy, positive prediction values, and negative prediction values are calculated to discriminate negative from positive samples, obtaining 100, 80, 90, 83.3, and $100 \%$, respectively. Raman spectroscopy, associated with the PLS, is promising as a serological assay for toxoplasmosis, enabling fast and sensitive diagnosis. ( 2010 Society of Photo-Optical Instrumentation Engineers. [DOI: 10.1117/1.3463006]
\end{abstract}

Keywords: near-infrared Raman spectroscopy; multivariate statistical analysis; partial least squares; toxoplasmosis; cats; serology; indirect enzyme-linked immunosorbent assay.

Paper 09457RR received Oct. 9, 2009; revised manuscript received Apr. 29, 2010; accepted for publication May 12, 2010; published online Jul. 21, 2010.

\section{Introduction}

Toxoplasmosis is a disease widely spread in many countries and it is caused by the Toxoplasma gondii protozoan. This disease presents a threat to the public health because the human infection by the protozoan is very common and can cause severe life-threatening diseases in immune compromised patients and in newborns with congenital toxoplasmosis. In addition $T$. gondii infection is important in veterinary medicine due to fetal abortions and neonatal losses of livestock. ${ }^{1}$ Toxoplasmosis transmission occurs by ingestion of sporulated oocysts released with the feces of infected cats and other felids or by cysts present in the tissues of an infected animal. Humans can be contaminated via congenital infection, fecal matter residue consumption, or ingestion of uncooked meat. ${ }^{2}$ Cats are the main cause for the toxoplasmosis transmission to hu-

Address all correspondence to: Landulfo Silveira Jr., Biomedical Engineerig Group, Universidade Camilo Castelo Branco-UNICASTELO, Núcleo do Parque Tecnológico de São José dos Campos, Rod. Pres. Dutra, km 138, São José dos Campos, SP, Brazil, 12247-004. Tel/Fax: 55-12-3905-4401; E-mail: landulfo.silveira@unicastelo.br or landulfo.silveira@gmail.com mans and other animals. ${ }^{3}$ The disease is found in several animal species that present important economic interest and it can cause severe profit losses if not detected.

Antibodies or immunoglobulins (Igs) are gamma globulin proteins that are found in blood or other body fluids of vertebrates, and they are used by the immune system to identify and neutralize foreign objects, such as bacteria and viruses. The diversity of antibodies enables the immune system to recognize an equally wide diversity of antigens. The unique part of the antigen recognized by an antibody is called an epitope. These epitopes bind with their antibodies in a highly specific interaction, called induced fit, that enables antibodies to identify and bind with only their unique antigen in the midst of the millions of different molecules that make up an organism. Specific IgM antibodies are the best indicators of a recent $T$. gondii infection in several species and can be detected from blood samples, indicating an early stage of the infection up to 12 weeks later, while $\mathrm{IgG}$ antibodies charac-

1083-3668/2010/15(4)/047002/7/\$25.00 @ $2010 \mathrm{SPIE}$ 
terize a chronic disease and may be present up to 52 weeks after the infection took place. ${ }^{4-6}$ Both antibodies can be used to detect several specific serum antigens with different infection levels as Neospora caninum, Leishmania spp, Histomonas meleagridis, and Cryptosporidium parvum, among others, and the antibody class investigation must be defined according to the study goal. $^{7-10}$

Enzyme-linked immunosorbent assay (ELISA) is the most widely used serological method to diagnose toxoplasmosis in domestic animals, since it provides a good sensitivity to detect IgG or IgM for T. gondii protozoan. ${ }^{11}$ Other serological assays in addition to ELISA are being used for IgG and IgM identification; for instance, chemiluminescense immunoassay (CLIA), enzyme-linkend fluorescent assay (ELFA), and indirect immunofluorescent assay (IFA). All of these provide similar sensitivities, specificities, and positive and negative predictive values. ${ }^{12}$ Nevertheless, these methods are not fast enough due to the necessity of sample preparation, including steps of washing, dilution, and incubation. Furthermore, the protein source used in the ELISA test usually contains multiple $T$. gondii antigens that may increase nonspecific crossreactivity providing false positive results. ${ }^{13}$ On the other hand, the use of other optical diagnosis techniques such as Fourier transform IR spectroscopy and near-IR Raman spectroscopy would enable a high sensitivity for molecular detection and they could be done faster and less expensively. Since they are nondestructive tests, there is no need for a sample preparation and, therefore, they can be automated. ${ }^{14}$ Riley et al. ${ }^{15}$ reported the use of the Fourier transform IR spectroscopy technique in conjunction to a partial least-squares (PLS) regression model to diagnose the failure of transfer of passive immunity in horses. They found sensitivity, specificity, accuracy, and predictive values for diagnosis as good as those obtained by using the radial immunodiffusion assay (RID).

The use of dispersive near-IR Raman spectroscopy has been expanding in the biomedical field. ${ }^{14,16,17}$ This vibrational technique could help to determine the molecular composition of materials and is capable of a fast, accurate, and real time biological diagnosis. ${ }^{14}$ Its use is being extended to the veterinary field. ${ }^{18,19}$ Raman spectroscopy is a useful tool for quantitative biological analysis as well as for clinical diagnosis in situ and ex situ, which can be used to reduce the risk of invasive procedures. ${ }^{20-22}$ It provides a high specificity to identify and quantify metabolites and pharmaceutical products ${ }^{23,24}$ and it has the potential to identify and characterize microorganisms (bacteria and fungi) rapidly. ${ }^{25-27}$ It can be used to determine the physiological conditions and the molecular composition of the tissues and organs through a noninvasive or minimally invasive method..$^{21,28-31}$ Torreggiani and Fini ${ }^{32}$ studied the connection between the protein avidin and biotin through Raman spectroscopy. This study indicated that Raman spectroscopy could be used to detect specific antibodies due to its high sensitivity for molecular detection. Application of Raman spectroscopy to detect the presence of antibodies against $T$. gondii in cat blood serum ${ }^{18}$ and in human colostrum has been reported. ${ }^{19}$

To be an effective and sensitive technique for biological material identification and classification, Raman spectroscopy requires an appropriate statistical method that can identify spectral differences that originate from differences in the sample composition that occur within the data set. Multivari- ate statistics frequently used for quantitative analysis in Raman spectroscopy are the PLS regression and principal component regression (PCR). Such techniques can promote a quantitative analysis of compounds presented in the samples, even in the presence of multiple components where multicollinearity cannot be avoided. It was very successful in quantifying biologically relevant analytes such as glucose, ammonia, glutamine, glutamate, and lactate diluted in complex mixtures. ${ }^{33}$ Berger et al. ${ }^{23}$ determined the concentrations of the metabolites glucose, cholesterol, triglycerides, urea, total protein, and albumin in human blood serum and human total blood by Raman spectroscopy and PLS regression. Recent applications of IR spectroscopy associated with PLS regression include a device for bedside monitoring of subcutaneous interstitial glucose using near-IR spectrometry, ${ }^{34}$ pulse glucometry for a noninvasive measurement of blood glucose, ${ }^{35}$ and determination of glucose in clinically relevant concentrations in artificial aqueous humor with Raman spectroscopy. ${ }^{36}$

The objective of this work was to develop a model for blood sera assay based on Raman spectroscopy and multivariate statistical analysis PLS regression, for the quantitative analysis of $T$. gondii antibody (IgG) in the sera of domestic cats without and with diagnosed toxoplasmosis that have been submitted to the quantitative evaluation of $\mathrm{IgG}$ by an indirect ELISA test, and to develop a diagnostic model for discriminating positive from negative sera. The ELISA assay is widely used for serology and was used as a gold standard for detecting IgG in the blood sera included in the PLS model. A preliminary study showing the potential of the dispersive near-IR (NIR) Raman spectroscopy technique for detecting T. gondii antibodies $(\mathrm{IgG})$ in cat blood sera was previously reported by our group,${ }^{18}$ indicating feasibility for such optical approach.

\section{Materials and Methods}

\subsection{Domestic Cat Blood Serum Samples}

A total of 59 cat blood serum samples were obtained from the Protection Animal Society (São Paulo, SP, Brazil) and used in the presented work. The samples were previously evaluated by the ELISA test, and following that, the Raman spectrum was taken for each sample.

\subsection{ELISA}

An ELISA test was performed at the Department of Pathology of Universidade Estadual Paulista (UNESP) (Jaboticabal, SP, Brazil) aiming to detect the presence of anti-T. gondii antibodies (IgG) in the cat serum. The ELISA test was performed following the protocol described by Domingues et al. ${ }^{37}$ Serological results were quantified and defined as a set of ELISA levels (between 0 and 9), corresponding to a range of the ELISA optical density (OD) of the samples, ${ }^{38}$ according to Table 1.

\subsection{Raman Spectroscopy}

A Raman spectrometer was used to collect the spectra; this instrument is described elsewhere. ${ }^{18,24}$ Briefly, the excitation source was a Ti:sapphire laser (Spectra Physics, model 3900S) pumped by a 6-W multiline argon laser (Spectra Physics, model 2709S), tuned to $830 \mathrm{~nm}$. The Raman scattered signal from the sample was collected and coupled into the spectrometer entrance slit by a set of lenses. The Rayleigh 
Table 1 OD by ELISA test, corresponding ELISA levels, and IgG serology.

\begin{tabular}{cccccc}
\hline ELISA OD Range & ELISA Level & IgG Serology & ELISA OD Range & ELISA Level & lgG Serology \\
\hline $0.000-0.221$ & 0 & $\mathrm{~N}$ & $0.736-0.993$ & 5 & $\mathrm{MP}$ \\
$0.222-0.298$ & 1 & $\mathrm{MP}$ & $0.994-1.340$ & 6 & $\mathrm{MP}$ \\
$0.299-0.403$ & 2 & $\mathrm{MP}$ & $1.341-1.809$ & 7 & $\mathrm{SP}$ \\
$0.404-0.544$ & 3 & $\mathrm{MP}$ & $1.810-2.443$ & 8 & $\mathrm{SP}$ \\
$0.545-0.735$ & 4 & $\mathrm{MP}$ & $2.444-3.298$ & 9 & $\mathrm{SP}$ \\
\hline
\end{tabular}

$(\mathrm{N}$, negative; MP, medium positive; and SP, strong positive).

scattering from the sample was strongly attenuated in the collecting system by an holographic notch filter (Kaiser Optical Systems, model HSPF 830AR2.0). The optical detection system was composed of a high coupling spectrograph (Kaiser Optical Systems, Inc., f/1.8i) and a liquid-nitrogen-cooled, deep depletion CCD camera and controller (Princeton Instruments, model EEV $1024 \times 256$ and model ST130, respectively). To collect the Raman signal with an optimal resolution, the spectrometer entrance slit width was set ${ }^{39}$ to $200 \mu \mathrm{m}$, with a spectral resolution of about $10 \mathrm{~cm}^{-1}$. The CCD controller was connected to a personal computer that stored and processed the Raman spectra.

The quartz cuvette with the serum sample was positioned facing the spectrometer entrance slit. The pumping laser beam impinged on the cuvette, forming a right angle with respect to the signal-collecting optical axis. The serum samples were irradiated with $80-\mathrm{mW}$ laser power and an acquisition time of $100 \mathrm{~s}$ for each sample.

The optical detection system (spectrometer and CCD) was calibrated in wave numbers using an organic compound, indene $\left(\mathrm{C}_{9} \mathrm{H}_{8}\right)$, which has a well-established Raman spectrum that presents intense and well-distributed bands covering the whole spectral region between 800 and $1800 \mathrm{~cm}^{-1}$.

\subsection{Preprocessing of NIR Raman Spectra}

The contribution of the fluorescence emission to the Raman spectra was eliminated by fitting and subtracting a third-order polynomial from the gross spectrum. After calibration and background subtraction, the Raman spectra were filtered using the MATLAB ${ }^{\circledR}$ software to reduce the high-frequency noise (associated with the shot and readout noise). Cosmic ray spikes were manually removed. Once filtered, spectra were normalized by the most intense band and submitted to a multivariate statistical analysis, composed of the PLS regression method.

\subsection{Multivariate Statistical Analysis_-PLS method}

Samples were classified according to their $\operatorname{IgG}$ serology into two groups for PLS analysis: negative and positive samples. Then the 59 preprocessed spectra were separated randomly in two groups: 49 were used as the calibration dataset and the remaining 10 as the validation (prediction) data set, including positive and negative samples in the calibration and validation groups. The PLS model was implemented by using the calibration set and the corresponding latent variables (LVs) were calculated from the first LV to the 10th LV.

To determine the number of LVs to be included in the PLS model that provides the minimum error predicted by the PLS compared to the ELISA OD values, we calculated the root mean square error of prediction (RMSEP) for each LV used in the validation data set as follows:

$$
\operatorname{RMSEP}=\left[\frac{\sum_{n}^{i=1}\left(c_{i}-c a_{i}\right)}{n}\right]^{1 / 2},
$$

where $c_{i}$ is the OD value predicted by the PLS for the sample $i, c a_{i}$ is the OD value measured by ELISA (the gold-standard technique), and $n$ is the number of samples. The minimum value of RMSEP will occur in the optimal LV, which can be used to predict future samples.

Likewise, the parameters sensitivity (Se), specificity (Sp), accuracy, positive predictive value (PPV), and negative predictive value (NPV) for the Raman/PLS model were calculated. Those parameters are defined as $\mathrm{Se}=\mathrm{TP} /(\mathrm{TP}+\mathrm{FN})$, $\mathrm{Sp}=\mathrm{TN} /(\mathrm{TN}+\mathrm{FP}), \quad$ accuracy $=(\mathrm{TP}+\mathrm{TN}) /(\mathrm{TP}+\mathrm{FP}+\mathrm{TN}$ $+\mathrm{FN}), \quad \mathrm{PPV}=\mathrm{TP} /(\mathrm{TP}+\mathrm{FP}), \quad$ and $\mathrm{NPV}=\mathrm{TN} /(\mathrm{FN}+\mathrm{TN})$, where TP, FP, TN, and FN are the numbers of true positives, false positives, true negatives, and false negatives, respectively.

\section{Results and Discussion}

\subsection{ELISA}

The 59 samples were classified as negative serum $(\mathrm{N})$, medium positive (MP) and strong positive (SP) according to the following criterion (Table 1): $\mathrm{N}$-ELISA level equal to 0 , MP-ELISA levels ranging from 1 to 6, and SP-ELISA levels above 6. Based on this classification, it was obtained $13 \mathrm{~N}, 30 \mathrm{MP}$, and $16 \mathrm{SP}$ blood sera.

\subsection{Raman Spectroscopy}

Figure 1 shows the Raman spectra of three characteristic samples with N, MP, and SP ELISA serologies in the spectral range between 900 and $1800 \mathrm{~cm}^{-1}$. All spectra have a similar profile, with prominent band positions at 944, 1003, 1058, $1169,1242,1331,1451$, and $1655 \mathrm{~cm}^{-1}$ for the three types of serology. An attempt to assign the bands presented in the serum, according to that reported by the literature, would be 


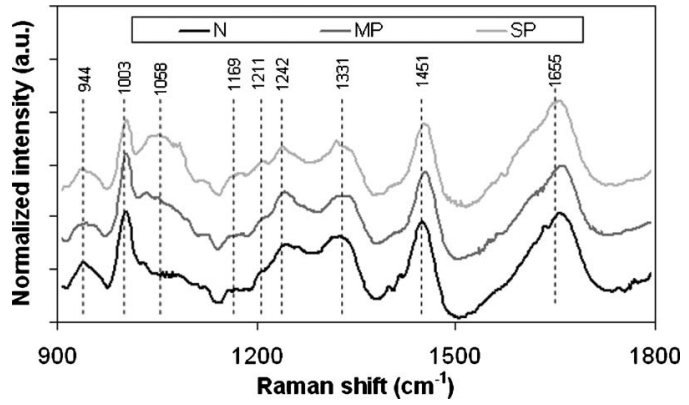

Fig. 1 Raman spectra of cat blood sera with the following serology to toxoplasmosis (IgG): negative $(\mathrm{N})$, medium positive (MP), and strong positive (SP).

band at $944 \mathrm{~cm}^{-1}$ (proteins: $\mathrm{C}-\mathrm{C}$ stretching mode of skeletal backbone $\alpha$-helix conformation); at $1003 \mathrm{~cm}^{-1}$ (phenylalanine: symmetric ring breathing mode); at $1058 \mathrm{~cm}^{-1}$ (phenylalanine and lipids: skeletal $\mathrm{C}-\mathrm{C}$ stretch); at $1169 \mathrm{~cm}^{-1}$ (amino acids and nucleic acids); at $1242 \mathrm{~cm}^{-1}$ (amide III from proteins: $\mathrm{N}-\mathrm{H}$ bending, $\mathrm{C}-\mathrm{N}$ stretch; $\mathrm{CH}_{2}$ wag); at $1331 \mathrm{~cm}^{-1}$ (proteins: $\mathrm{CH}_{3}$ and $\mathrm{CH}_{2}$ wagging; polynucleotide chain; phospholipids); at $1451 \mathrm{~cm}^{-1}$ (lipids and proteins: $\mathrm{CH}_{2}$ deformation and proteins: $\mathrm{CH}_{2}$ bending); and at $1655 \mathrm{~cm}^{-1}$ (lipids: $\mathrm{C}=\mathrm{C}$ stretching and amide $\mathrm{I}$ from proteins: $\mathrm{C}=\mathrm{O}$ stretching $\alpha$-helix conformation). ${ }^{14,16,40,41}$ Differences observed in the band location compared to literature are within the spectrometer resolution $\left(<10 \mathrm{~cm}^{-1}\right)$. The overall spectrum profiles are very similar for negative and positive sera; however, it was possible to observe Raman bands with higher intensities for the MP and SP samples when compared to the $\mathrm{N}$ samples, which could be explained by the presence of IgG antibodies. For instance, the bands at $1242 \mathrm{~cm}^{-1}$ (proteins) and $1058 \mathrm{~cm}^{-1}$ (lipids) have significant differences, increasing with disease gravity.

\subsection{PLS Model}

The PLS regression model was used to develop a method to obtain the equivalent OD for the Raman spectra, thus obtaining an OD quantification and serology identification for the Raman/PLS model of cat blood sera. The data set was divided into two groups: 49 spectra for training the model and 10 spectra for validation (prediction), including negative and positive sera in both groups. First, we developed the calibration curve with the 49 spectra, in which the OD obtained by the ELISA test were used to determine the equivalent OD predicted by Raman/PLS. To determine how many LVs should be included in the PLS model to give the lowest prediction error, the 10 spectra from the validation data set were submitted to the PLS model using the number of LVs from 1 to 10 . Then the RMSEP parameter was calculated for the OD values of the validation data set (Figure 2). We found that a lower RMSEP parameter is reached using seven LVs, so the OD values calculated using seven LVs for both training and validation groups versus the OD by ELISA are plotted in Fig. $3(\mathrm{a})$, whereas that for the validation group alone, separated by serology type, are shown in Fig. 3(b). We observed that all points lie close to the diagonal, zero-error line. The prediction RMSEP had a value of about $\mathrm{OD}=0.18$, which is satisfactory

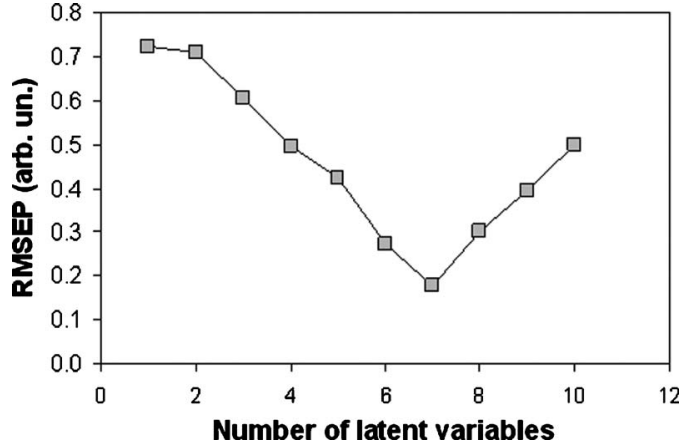

Fig. 2 Plot of the RMSEP values of the OD predicted by the Raman/ PLS model compared to OD values by ELISA using the first $10 \mathrm{LVs}$ of the validation Raman data set.

for a method of rapid serology assay considering that the upper cutoff for the negative serology was $\mathrm{OD}=0.221$.

\subsection{Diagnosis Analysis}

A comparison between OD values obtained by the Raman/ PLS regression model and those obtained by the ELISA test in the validation data set is shown in Table 2. The table also shows the Se, Sp, accuracy, and PPV and NPV for the validation data set, considering positive the sera with OD $>0.221$. We did not observe any false negative diagnosis, although we found only one sample out of the 10 analyzed that was identified as negative by the ELISA and diagnosed as positive by the Raman/PLS method. With respect to this
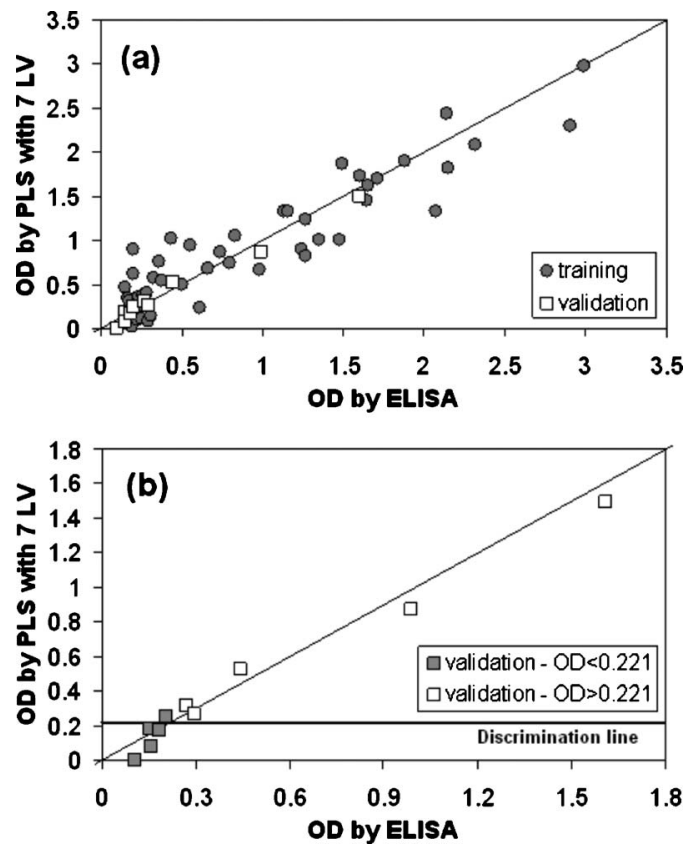

Fig. 3 (a) Plot of the OD values predicted by the Raman-PLS model using seven LVs applied to cat blood sera versus the OD values obtained by ELISA test. Training group: 49 spectra, validation group: 10 spectra. The diagonal line represents the zero-error prediction line. (b) Plot of the OD achieved by the Raman/PLS model versus the OD from the ELISA test on a scale of 0 to 1.8 of the validation data set. The horizontal line represents $\mathrm{OD}=0.221$, which discriminates negative from positive $\lg \mathrm{G}$ serologies. 
Table 2 Classification capabilities of the Raman/PLS diagnosis correlated to the ELISA test (OD values) for the IgG from serum of domestic cats of the validation spectra.

\begin{tabular}{|c|c|c|c|c|c|c|c|c|}
\hline \multirow{2}{*}{$\begin{array}{c}\text { Raman-PLS } \\
\text { Diagnosis } \\
\text { Validation } \\
\text { data set }\end{array}$} & \multicolumn{8}{|c|}{ ELISA } \\
\hline & $O D \leqslant 0.221$ & $O D>0.221$ & Total & $\mathrm{Se}(\%)$ & $\mathrm{Sp}(\%)$ & Accuracy (\%) & PPV (\%) & NPV (\%) \\
\hline $\begin{array}{c}\text { ELISA OD } \leqslant \\
0.221\end{array}$ & 4 & 0 & 4 & & & & & \\
\hline $\begin{array}{c}\text { ELISA OD> } \\
0.221\end{array}$ & 1 & 5 & 6 & 100 & 80 & 90 & 83.3 & 100 \\
\hline Total & 5 & 5 & 10 & & & & & \\
\hline
\end{tabular}

Se, sensitivity; Sp, specificity; PPV, positive predictive value; NPV, negative predictive value.

sample, it was verified that this misclassified sample presented an OD value that is very close to the upper cutoff value for negative serum, and, therefore, the prediction seems to be more uncertain.

Riley et al. studied the diagnosis of the failure of transfer of passive immunity in horses using Fourier transform (FT) infrared spectroscopy employing PLS regression analysis. ${ }^{15}$ They found parameter values of about 92, 96, 95, 86, and $98 \%$, for Se, Sp, accuracy, PPV, and NPV, respectively. To compare Riley et al.'s work and the presented study, one should notice that the meaning of negative and positive is different in these works: FPT-positive in the Riley et al. paper is equivalent to serum-negative for toxoplasmosis in this study and vice versa. Therefore, the meanings of Se, Sp, PPV, and NPV are apposite in these works. These values are com- parable to the ones presented in this model for toxoplasmosis diagnosis using Raman/PLS regression analysis (Table 2).

Table 3 compares the capabilities of the dispersive Raman spectroscopy in conjunction with a multivariate statistical analysis in diagnostic models for different diseases as reported in the literature. ${ }^{42-45}$ We can see that our results on toxoplasmosis identification agree well with those obtained for other diseases using the same spectroscopic technique.

The NIR Raman technique has been proven to be suitable for biotissue analysis (including tissues, blood, and serum) due to the intrinsic vibrational information that is carried out by the Raman signal without interference from water. By using Raman spectroscopy, usually no sample preparation is required, a small amount of sample is used, the biochemical alterations could be obtained directly in real time, and de-

Table 3 Comparison of the diagnostic capabilities of the Raman spectroscopy associated with multivariate statistical analysis in the diagnosis of several diseases.

\begin{tabular}{|c|c|c|c|}
\hline Diagnostic Test for & Spectroscopic Method & $\mathrm{Se}(\%)$ & $\mathrm{Sp}(\%)$ \\
\hline Oral carcinoma ${ }^{a}$ & FT-Raman & 100 & 55 \\
\hline Hepatitis $C^{b}$ & NIR dispersive Raman & 92 & 88 \\
\hline Atherosclerosis in coronary artery ${ }^{c}$ & NIR dispersive Raman & & \\
\hline Nonatherosclerotic plaque & & 78 & 93 \\
\hline Noncalcified plaque & & 90 & 83 \\
\hline Calcified plaque & & 100 & 100 \\
\hline Atherosclerosis in carotid artery ${ }^{d}$ & FT-Raman & & \\
\hline Nonatherosclerotic plaque & & 96 & 93 \\
\hline Atherosclerotic plaque noncalcified & & 89 & 96 \\
\hline Atherosclerotic plaque calcified & & 100 & 100 \\
\hline Toxoplasmosis ${ }^{\mathrm{e}}$ & NIR dispersive Raman & 100 & 80 \\
\hline $\begin{array}{l}\text { वOliveira et al. }{ }^{42} \\
{ }^{4} \text { Saade et al }{ }^{43} \\
{ }^{\circ} \text { Silveira et al. }{ }^{44} \\
{ }^{d} \text { Nogueira et al. }{ }^{45} \\
\text { eThis work }\end{array}$ & & & \\
\hline
\end{tabular}


pending on the biochemical of interest, it results in a unique molecular fingerprint, which can be used to discriminate qualitatively and quantitatively the diseased tissue. Recent developments in Raman instrumentation include the use of lowcost diode lasers, CCD detectors with electronic cooling, fiber optic probes designed to reduce fiber background and increase SNR, and compact spectrometers with high throughput. ${ }^{46}$

\section{Conclusions}

Results indicated that the dispersive NIR Raman spectroscopy method in conjunction with a PLS regression analysis (Raman/PLS model) is effective in detecting toxoplasmosis in blood sera of domestic cats compared to the ELISA test. This method was shown to be very promising as an alternative technique for toxoplasmosis diagnosis, providing several advantages over conventional serological methods of diagnosis.

\section{Acknowledgments}

J. Duarte acknowledges FAPESP (Fundação de Amparo à Pesquisa do Estado de São Paulo) for the graduate scholarships. L. Silveira Jr. thanks CNPq (Conselho Nacional de Desenvolvimento Científico e Tecnológico) for the Productivity Fellowship (305610/2008-2).

\section{References}

1. H. Caballero-Ortega, H. Quiroz-Romero, S. Olazara, N. Jenkins, and D. Correa, "Frequency of Toxoplasma gondii infection in sheep from a tropical zone of Mexico and temporal analysis of the humoral response changes," Parasitology 135, 897-902 (2008).

2. J. P. Dubey, "Toxoplasmosis - a waterborne zoonosis," Vet. Parasitol. 126, 57-72 (2004).

3. V. Svobodova, Z. Knotek, and M. Svoboda, "Prevalence of IgG and IgM antibodies specific to Toxiplasma gondii in rats," Vet. Parasitol. 80, 173-176 (1998).

4. M. R. Lappin, C. E. Greene, A. K. Prestwood, D. L. Dawe, and R. L. Tarleton, "Enzime linked immunosorbent assay for the detection of circulating antigens of Toxoplasma gondii in the serum of cats," Am. J. Vet. Res. 50, 1586-1590 (1989).

5. M. R. Lappin, C. E. Greene, A. K. Prestwood, D. L. Dawe, and R. L. Tarleton, "Diagnosis of recent Toxoplasma gondii infection in cats by use of an enzyme linked immunosorbent assay for immunoglobulin M," Am. J. Vet. Res. 50, 1580-1585 (1989).

6. J. Cozon, J. Ferrandiz, H. Nebhi, M. Wallon, and F. Peyron, "Estimation of the avidity of immunoglobulin $\mathrm{G}$ for routine diagnosis of chronic Toxoplasma gondii infection in pregnant women," Eur. J. Clin. Microbiol. Infect. Dis. 17, 32-36 (1998).

7. J. P. Dubey and G. Schares, "Diagnosis of bovine neosporosis," Vet. Parasitol. 140, 1-34 (2006).

8. L. M. Garcia, J. G. A. Coelho-dos-Reis, V. Peruhype-Magalhães, A Teixeira-Carvalho, R. D. R. Rocha, M. S. S. Araújo, I. T. Gomes, S F. G. Carvalho, R. Dietze, E. M. Lemos, and O. A. Martins-Filho, "Anti-fixed Leishmania chagasi promastigotes IgG antibodies detected by flow cytometry (FC-AFPA-IgG) as a tool for serodiagnosis and for post-therapeutic cure assessment in American visceral leishmaniasis," J. Immunol. Methods 350, 36-45 (2009).

9. M. Windisch and M. Hess, "Experimental infection of chickens with Histomonas meleagridis confirms the presence of antibodies in different parts of the intestine," Parasite Immunol. 32, 29-35 (2010)

10. K. Kaushik, S. Khurana, A. Wanchu, and N. Malla, "Serum immunoglobulin G, M and A response to Cryptosporidium parvum in Cryptosporidium-HIV co-infected patients," BMC Infect. Dis. 9, 179 (2009).

11. D. Buxton, "Protozoan infections (Toxoplasma gondii, Neospora caninum and Sarcocystis spp.) in sheep and goats: recent advances," Vet. Res. 29, 289-310 (1998).
12. M. J. Gharavi, H. Oormazdi, and E. S. Roointan, "A comparative study on sensitivity and specificity of conventional and unconventional IgG and IgM assays for diagnosis of Toxoplasmosis," Iranian J. Publ. Health 37, 42-45 (2008).

13. M. M. Conde de Felipe, J. M. Molina, E. Rodríguez-Ponce, A. Ruiz, and J. F. González, "IgM and IgG response to 29-35-kDa Toxoplasma gondii protein fractions in experimentally infected goats," $J$. Parasitol. 93, 701-703 (2007).

14. E. B. Hanlon, R. Manoharan, T. W. Koo, K. E. Shafer, J. T. Motz, M. Ftzmaurices, J. R. Kramer, I. Itzkan, R. R. Dasari, and M. S. Feld, "Prospects for in vivo Raman spectroscopy," Phys. Med. Biol. 45, R1-R59 (2000).

15. C. B. Riley, J. T. McClure, S. Low-Ying, and R. A. Shaw, "Use of Fourier transform infrared spectroscopy for the diagnosis of failure of passive transfer of immunoglobulin in horses," J. Vet. Intern Med. 21 , 828-834 (2007).

16. R. Manoharan, Y. Wang, and M. S. Feld, "Histochemical analysis of biological tissues using Raman spectroscopy," Spectrochim. Acta, Part A 52, 215-249 (1996).

17. R. Manoharan, J. J. Baraga, M. S. Feld, and R. P. Rava, "Quantitative histochemical analysis of human artery using Raman spectroscopy," J. Photochem. Photobiol., B 16, 211-233 (1992).

18. J. Duarte, M. T. T. Pacheco, R. Z. Machado, L. Silveira Jr., R. A. Zângaro, and A. B. Villaverde, "Use of near-infrared Raman spectroscopy to detect IgG and IgM antibodies against Toxoplasma gondii in serum samples of domestic cats," Cell. Mol. Biol. 48, 585-589 (2002).

19. C. Araujo-Andrade, J. L. Pichardo-Molina, G. Barbosa-Sabanero, C. Frausto-Reyes, and A. Torres-López, "Detection of the presence of antibodies against Toxoplasma gondii in human colostrum by Raman spectroscopy and principal component analysis," J. Biomed. Opt. 12 , 034006 (2007)

20. C. Mello, K. J. Ciuffi, E. Nassar, D. Ribeiro, and R. J. Poppi, "Twodimensional low resolution Raman spectroscopy applied to fast discrimination of clinically relevant microorganisms: a whole-organism fingerprinting approach," J. Braz. Chem. Soc. 17(1), 73-78 (2006).

21. L. M. Moreira, L. Silveira Jr., F. V. Santos, J. P. Lyon, R. Rocha, R. A. Zângaro, A. Balbin Villaverde, and M. T. T. Pacheco, "Raman spectroscopy: a powerful technique for biochemical analysis and diagnosis," Spectrosc. Int. J. 22, 1-19 (2008).

22. M. G. Shim, L. M. W. K. Song, N. E. Marcon, and B. C. Wilson, "In vivo near-infrared Raman spectroscopy: demonstration of feasibility during clinical gastrointestinal endoscopy," Photochem. Photobiol. 72(1), 146-150 (2000).

23. A. J. Berger, T. W. Koo, I. Itzkan, G. Horowitz, and M. S. Feld, "Multicomponent blood analysis by near-infrared spectroscopy," Appl. Opt. 38, 2916-2926 (1999).

24. S. Pilotto, M. T. T. Pacheco, L. Silveira, Jr., A. B. Villaverde, and R. A. Zângaro, "Analysis of near-infrared Raman spectroscopy as a new technique for a transcutaneous noninvasive diagnosis of blood components," Lasers Med. Sci. 16, 2-9 (2001).

25. M. Harz, P. Rösch, and J. Popp, "Vibrational spectroscopy-a powerful tool for the rapid identification of microbial cells at the singlecell level," Cytometry, Part A 75, 104-113 (2009).

26. M. Krause, P. Rosch, B. Radt, and J. Popp, "Localizing and identifying living bacteria in an abiotic environment by a combination of Raman and fluorescence microscopy," Anal. Chem. 80, 8568-8575 (2008).

27. K. Maquelin, C. Kirschner, L. P. Choo-Smith, N. A. Ngo-Thi, T. van Vreeswijk, M. Stämmler, H. P. Endtz, H. A. Bruining, D. Naumann, and G. J. Puppels, "Prospective study of the performance of vibrational spectroscopies for rapid identification of bacterial and fungal pathogens recovered from blood cultures," J. Clin. Microbiol. 41, 324-329 (2003).

28. P. J. Caspers, G. W. Lucassen, E. A. Carter, H. A. Bruining, and G. J. Puppels, "In vivo confocal Raman microspectroscopy of the skin: non-invasive determination of molecular concentration profiles," $J$. Invest. Dermatol. 116, 434-442 (2001).

29. T. Bocklitz, M. Putsche, C. Stuber, J. Kaes, A. Niendorf, P. Rosch, and J. Popp, "A comprehensive study of classification methods for medical diagnosis," J. Raman Spectrosc. 40, 1759-1765 (2009).

30. P. Stavroulaki, N. Stone, C. Fulljames, H. Barr, and M. Birchall, "Raman spectroscopy for early detection of laryngeal malignancy: preliminary results," Clin. Otolaryngol. Allied Sci. 25, 91-92 (2000).

31. R. Rocha, L. Silveira Jr., A. Balbin Villaverde, C. A. Pasqualucci, M. 
S. Costa, A. Brugnera Jr., and M. T. T. Pacheco, "Use of near-infrared Raman spectroscopy for identification of atherosclerotic plaques in the carotid artery," Photomed. Laser Surg. 25, 480-484 (2007).

32. A. Torreggiani and G. Fini, "Raman spectroscopic studies of ligandprotein interactions: the binding of biotin analogues by avidin," $J$. Raman Spectrosc. 29, 229-236 (1998).

33. J. M. McShane, G. L. Coté, and C. H. Spielgelman, "Assessment of partial least-squares calibration and wavelength selection for complex near-infrared spectra," Appl. Spectrosc. 52, 878-884 (1998).

34. H. M. Heise, U. Damm, M. Bodenlenz, V. R. Kondepati, G. Koehler, and M. Ellmerer, "Bedside monitoring of subcutaneous interstitial glucose in healthy individuals using microdialysis and infrared spectrometry," J. Biomed. Opt. 12, 024004 (2007).

35. K. Yamakoshi and Y. Yamakoshi, "Pulse glucometry: a new approach for noninvasive blood glucose measurement using instantaneous differential near-infrared spectrophotometry," J. Biomed. Opt. 11, 054028 (2006).

36. J. L. Lambert, C. C. Pelletier, and M. Borchert, "Glucose determination in human aqueous humor with Raman spectroscopy," J. Biomed. Opt. 10, 031110 (2005).

37. L. M. Domingues, R. Z. Machado, M. T. Costa, C. S. Carvalho, A. J. Costa, and E. B. Malheiros, "Canine toxoplasmosis: a comparative evaluation of the detection of anti-Toxoplasma gondii antibodies by the indirect immunoenzymatic assay (ELISA) and indirect immunofluorescence reaction (IIF)," Braz. J. Vet. Parasitol. 7, 79-85 (1998).

38. R. Z. Machado, H. J. Montassier, A. A. Pinto, E. G. Lemos, M. R. F Machado, I. F. F. Valadão, L. G. Barci, and E. B. Malheiros, "An enzyme-linked immunosorbent assay (ELISA) for the detection of antibodies against Babesia bovis in cattle," Vet. Parasitol. 71, 17-26 (1997).
39. J. C. Lázaro, M. T. T. Pacheco, K. C. Rodrigues, C. J. Lima, L. M. Moreira, A. B. Villaverde, and L. Silveira, Jr., "Optimizing the Raman signal for characterizing organic samples: the effect of slit aperture and exposure time," Spectrosc. Int. J. 23, 71-80 (2009).

40. N. Stone, C. Kendall, J. Smith, P. Crow, and H. Barr, "Raman spectroscopy for identification of epithelial cancers," Faraday Discuss. 126, 141-157 (2004).

41. J. L. Pichardo-Molina, C. Frausto-Reyes, O. Barbosa-García, R. Huerta-Franco, J. L. González-Trujillo, C. A. Ramírez-Alvarado, G. Gutiérrez-Juárez, and C. Medina-Gutiérrez, "Raman spectroscopy and multivariate analysis of serum samples from breast cancer patients," Lasers Med. Sci. 22, 229-236 (2007).

42. A. P. Oliveira, R. A. Bitar, L. Silveira, Jr., R. A. Zângaro, and A. A. Martin, "Near-infrared Raman spectroscopy for oral carcinoma diagnosis," Photomed. Laser Surg. 24, 348-353 (2006).

43. J. Saade, M. T. T. Pacheco, M. R. Rodrigues, and L. Silveira Jr., "Identification of hepatitis $\mathrm{C}$ in human blood serum by near-infrared Raman spectroscopy," Spectrosc. Int. J. 22, 387-395 (2008).

44. L. Silveira Jr., S. Sathaiah, R. A. Zângaro, M. T. T. Pacheco, M. C. Chavantes, and C. A. G. Pasqualucci, "Correlation between nearinfrared Raman spectroscopy and the histopathological analysis of Atherosclerosis in human coronary arteries," Lasers Surg. Med. 30 , 290-297 (2002).

45. G. V. Nogueira, L. Silveira, Jr., A. A. Martin, R. A. Zângaro, M. T. T. Pacheco, M. C. Chavantes, and C. A. Pasqualucci, "Raman spectroscopy study of atherosclerosis in human carotid artery," J. Biomed. Opt. 10, 031117 (2005).

46. J. Zhao, H. Lui, D. I. McLean, and H. Zeng, "Integrated real-time Raman system for clinical in vivo skin analysis," Skin Res. Technol. 14, 484-492 (2008). 